\title{
Reconfigurable Surface Design for Electromagnetic Wave Control
}

\author{
Fereshteh. Samadi, and Abdelrazik. Sebak \\ Electrical and Computer Department, Concordia University, Montreal, Quebec H3G 1M8, Canada \\ F_samadi@encs.concordia.ca, abdo@ece.concorida.ca
}

\begin{abstract}
A reconfigurable surface, for electromagnetic (EM) wave manipulation, utilizing frequency selective unit-cells integrated with PIN diodes are introduced. By digitally controlling the reconfigurable surface, several functionalities such as focusing, linear polarization conversion and scattering are obtained on the same surface. For the scattering purposes, three arrangement methods of alternative periodic columns, chessboard-like structure and coding surface optimized by GSO algorithm are analyzed. For the coded surface, 10-dB RCS reduction from $15.5 \mathrm{GHz}$ to $22.5 \mathrm{GHz}$ is obtained with a bandwidth of $37 \%$. All the results show that the introduced reconfigurable surface has more potential for future applications. Keywords-Active frequency selective surface (FSS), reconfigurable surface, scattering, polarization conversion, security building, focusing.
\end{abstract}

\section{INTRODUCTION}

Frequency selective surfaces (FSSs), normally consist of artificially periodic or quasi-periodic structures, which provide several design strategies for various functionalities. They have been applied in several applications such as reducing interference [1], minimizing radar cross section (RCS) [2] and shielding [3] to name a few. Most of the conventional designs were focused on a certain function where the EM wave manipulation was fixed for the designed prototype. Therefore, conventional FSSs showed a limitation when employed to modern multifunctional and complex systems. To address this problem, a large amount of studies has been devoted to developing tunable FSS whose operation status can be controlled and subsequently, flexible functionalities can be achieved. The ability to electrically tune or alter the functionality of a surface has been realized through loading with active semiconductor devices, such as varactors and PIN diodes. They have been employed in several practical applications like reconfigurable antennas [4], designing intelligent walls [5]- [9] and radomes [10], [11]. Nowadays, one of the appealing applications of reconfigurable surfaces is a programmable wireless environment [12]. In this application, electromagnetic (EM) waves can be effectively engineered for several purposes including steering toward any desired direction, scattering, polarization manipulation and more.

In this paper, we present a tunable surface for multifunctional applications such as programmable wireless environment, see Fig. 1. The proposed surface is constructed of active unit-cells loaded by PIN diodes. Since each unit-cell can be controlled independently, the surface has ability to digitally control EM waves for versatile functions such as scattering, focusing, steering and polarization conversion. For the scattering purposes, $180^{\circ} \pm 37^{\circ}[13]$ reflection phase difference between ON and OFF states of the unit-cells in a frequency range of $16.25 \mathrm{GHz}$ to $22.75 \mathrm{GHz}$ is realized. To find the optimal ON and OFF states of the unit-cells, optimization algorithm of group search optimization (GSO) is applied, where very low RCS with a bandwidth of $37 \%$ (frequency range between $15.5 \mathrm{GHz}$ to $22.5 \mathrm{GHz}$ ) is achieved. Other functionalities are also proved through simulations which clearly shows the applicability of the proposed work for multifunctional systems such as the aforementioned programmable wireless environment.

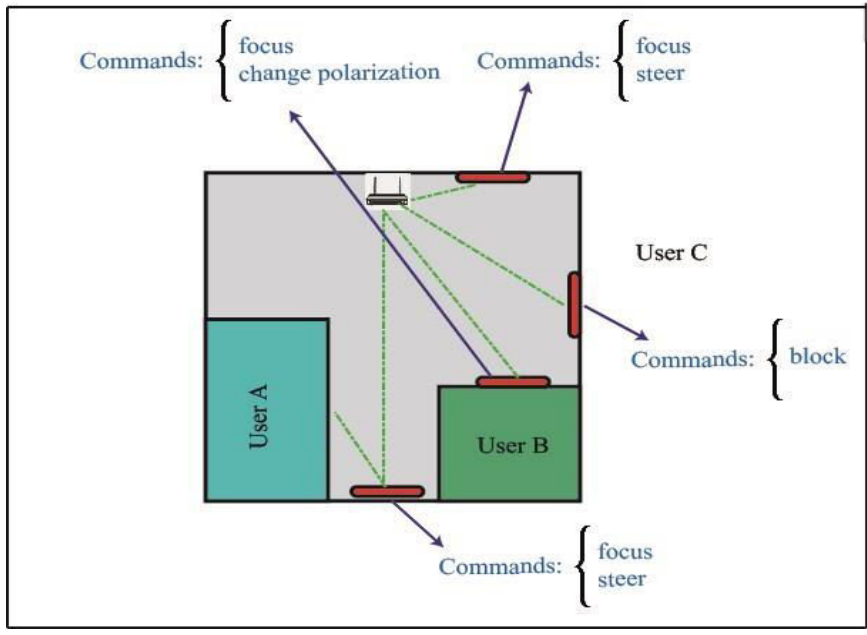

Fig. 1. Programmable wireless environment with an example of commands to manipulate EM waves to match the requirements of users.

\section{ACTIVE FSS DESIGN}

The geometrical configuration of the proposed multi-functional FSS unit-cell is depicted in Fig. 2 (a,b). The patch is mounted on Arlon diclad 880 , with permittivity of 2.2 and thickness of $1.542 \mathrm{~mm}$. To have reflection type unit-cell, a uniform ground plane is selected. In addition MACOM MADP-000907-14020 PIN diode is loaded on the 
top to connect the patch to the ground plane through a metal via. The $\mathrm{PIN}$ diode is modeled as equivalent series resistance and inductance of $7.8 \Omega$ and $30 \mathrm{pH}$, when the diode is $\mathrm{ON}$, and series capacitance and inductance $7.8 \Omega$ and $28 \mathrm{pF}$, when it is OFF, see Fig. 2, (c). The reflection phases for both $\mathrm{ON}$ and OFF conditions are investigated through HFSS software and shown in Fig. 3. The reflection phase difference between ON and OFF conditions is also shown in Fig. 3. Considering Fig. 3 , the $180^{\circ} \pm 37^{\circ}[13]$ reflection phase difference is ranging from $16.25 \mathrm{GHz}$ to $22.75 \mathrm{GHz}$, with a $33 \%$ frequency bandwidth, which is quite wide for active FSS unit-cell.

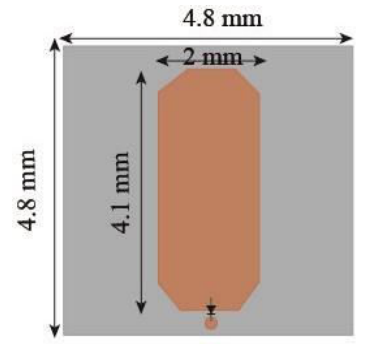

(a)

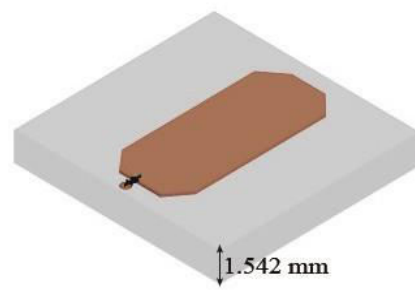

(b)

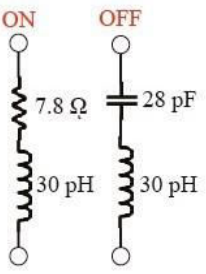

(c)

Fig. 2. Geometrical configuration of proposed active FSS unit-cell loaded by PIN diode. (a) Front view, (b) Side view, (c) The equivalent circuit of PIN diode.

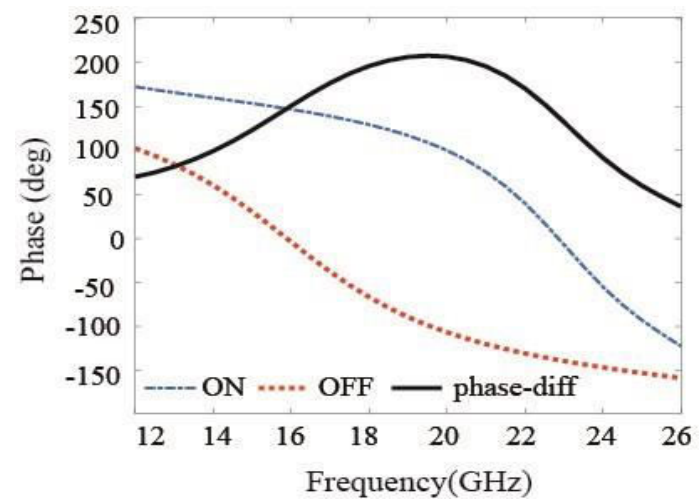

Fig. 3. Reflection phase coefficient, ON state, OFF state and the phase difference between two states.

\section{RESUlTS FOR MULTIPLE FUNCTIONALTIES}

After designing the unit-cell, in order to satisfy periodicity $4 \times 4$ unit-cells are considered as a tile. The ON and OFF states are mimicked as 1 and 0 digits. The overall dimension of 192 $\mathrm{mm} \times 192 \mathrm{~mm}$ consisting of $10 \times 10$ tiles are used. The multifunctionality is achieved by simply varying 0 and 1 , i.e., changing $\mathrm{ON}$ and OFF states of the tiles. We will investigate every functionalitiy in the following section.

\section{A. Linear Polarization Conversion}

Linear polarization conversion can be attained when all the unit-cells in the surface work at ON or OFF states simultaneously, which means all ' 1 ' or ' 0 ' coding matrices. In order to analyze the polarization conversion efficiency, the surface is illuminated by a diagonal plane wave, as it is shown in Fig. 4(a). The cross-and co-reflection amplitudes are simulated using HFSS software. As it can be seen from Fig. 4 (b), in the frequency range of $12 \mathrm{GHz}$ to $21 \mathrm{GHz}$, the crossreflected amplitude is around $0 \mathrm{~dB}$, however; the co-one is very low around $-10 \mathrm{~dB}$ with a peak of $-35 \mathrm{~dB}$. This shows the ability of the structure to rotate the polarization.

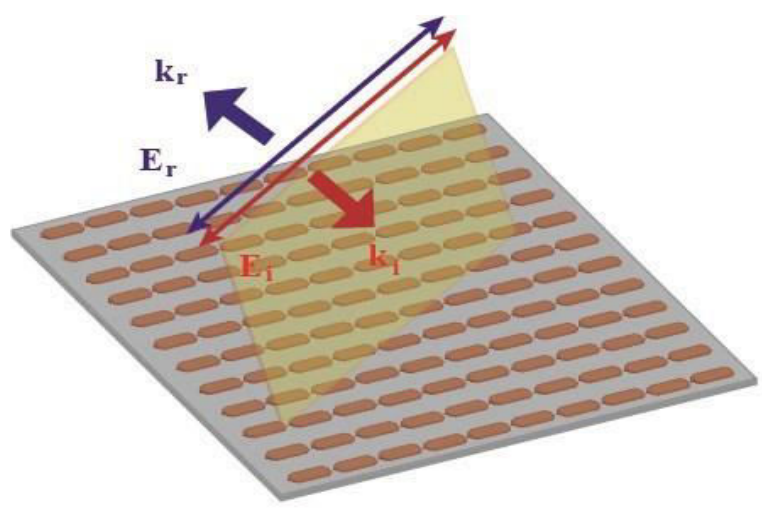

(a)

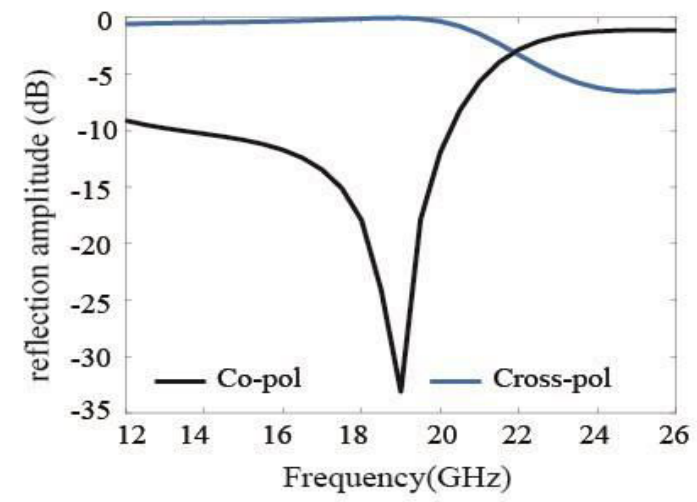

(b)

Fig. 4. Linear polarization conversion. (a) Schematic view of surface illuminated by diagonal plane wave, (b) Simulated co-and cross reflection amplitudes.

\section{B. Focusing}

When all unit-cells are OFF or ON, focusing properties can be achieved. Fig. 5 shows the reflected focused pattern from the surface when all diodes are ON. In this paper, the corresponding focused beam pointed at $0^{\circ}$, however; the pattern 
can be steered in other directions as well, by adjusting phase wave-front.

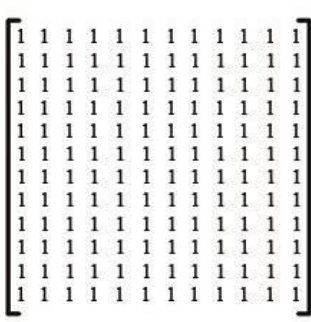

(a)

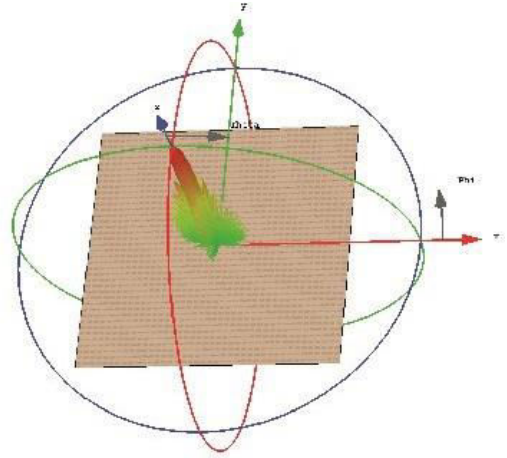

(b)
Fig. 5. Focusing properties for the case that all unit-cells are ON.

\section{Scattering properties}

Various scattering pattern can also be achieved when the surface is illuminated by a plane wave. To do so, several coding matrices are analyzed. Firstly, ' 1 ' and ' 0 ' tiles are arranged alternatively. This arrangement results in two main beams, see Fig. 6 (a,b). Afterward, a chess-board binary coding configuration shown in Fig. 6(c) is applied, and as it is expected four main lobes are achieved when illuminated by an incident plane wave. Finally, to attain a very uniform scattered pattern, the coding matrix is obtained applying GSO [14] optimization algorithm. Fig. 6 (e,f) depicts the optimal coding matrix and the scattered pattern from the optimal surface. As can be seen from the scattered pattern, very uniform pattern with the lowest maximum RCS is achieved for the coded surface. To further investigate the RCS reduction properties for the three designs, the monostatic normalized RCS is simulated and shown in Fig. 7. It worth mentioning that, the surfaces are normalized by the same size PEC plane. Comparing the RCS reduction curves in Fig. 7, it can be realized that the optimal surface contributes to the widest bandwidth and the lowest RCS reduction. The bandwidth for the coded surface is about $37 \%$, ranging from $15.5 \mathrm{GHz}$ to $22.5 \mathrm{GHz}$. This value is in agreement with the bandwidth of the reflection phase difference in Fig. 3, as it is expected.

\section{CONCLUSION}

We presented a multifunctional reconfigurable structure with the bandwidth of $37 \%$ ranging from $15.5 \mathrm{GHz}$ to 22.5 $\mathrm{GHz}$. The proposed design switches between polarization convertor, scattering and focusing surface. The proposed FSS structure consists of 10 by 10 tiles, where by deliberately switching every tile the desired functionality is achieved. The powerful manipulating ability of the EM wave indicates that the proposed FSS structure has great promise for programmable wireless environments and intelligent walls.

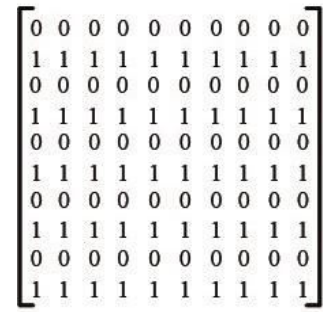

(a)

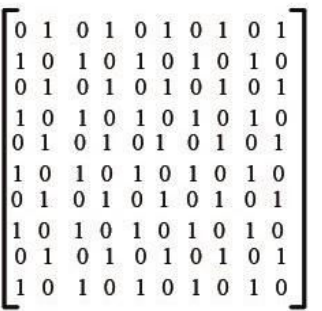

(c)

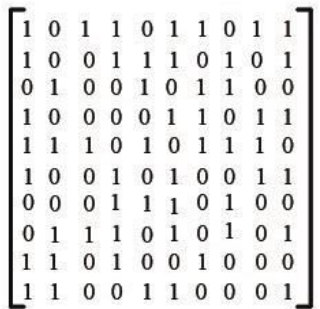

(e)

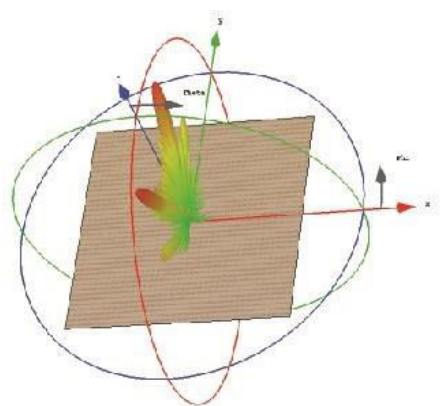

(b)

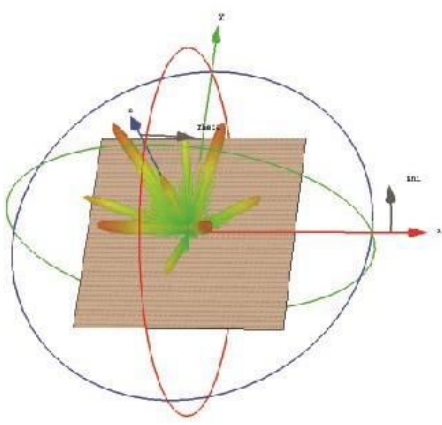

(d)

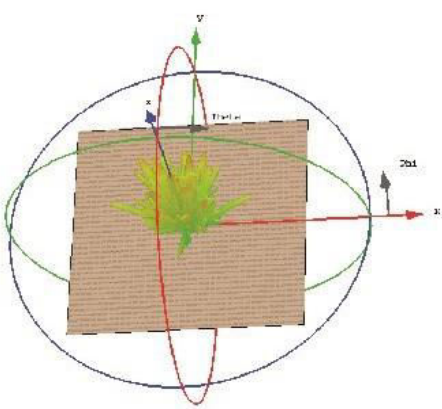

(f)
Fig. 6. Various scattering properties under normal incidence wave. $(a, b)$ Alternative structure matrix and associated scattered pattern, (c,d) Chessboardlike structure matrix and associated scattered pattern, $(\mathrm{e}, \mathrm{f})$ coded structure matrix and associated scattered pattern.

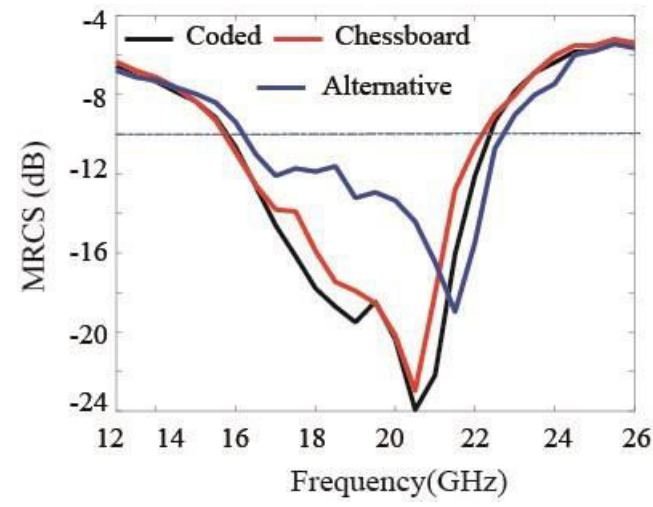

Fig. 7. Monostatic normalized RCS reduction. 


\section{REFERENCES}

[1] M. Yan, S. Qu, J. Wang et al., "A miniaturized dual-band FSS with stable resonance frequencies of $2.4 \mathrm{GHz} / 5 \mathrm{GHz}$ for WLAN applications," IEEE Antennas and Wireless Propagation Letters, vol. 13, pp. 895-898, 2014.

[2] C. Sudhendra, A. R. Madhu, A. C. R. Pillai, R. Kark, and T. S. Rukmini, "A novel ultra wide band radar absorber based on hexagonal resistive patch FSS," in Proceedings of the $4^{\text {th }}$ IEEE Applied Electromagnetics Conference, AEMC 2013, pp. 1-2, December 2013.

[3] R. Sivasamy, M. Lingeshwaran, K. Malathi, S. Esther Florence, and A. M. Gulam Nabi, "A low-profile paper substrate-based dual-band FSS for GSM shielding," IEEE T ELECTROMAGN C, vol. 58, no. 2, 2016, pp. 611-614.

[4] Sanz-Izquierdo, B., Liang, B., Parker, E.A., et al., "An application of active frequency selective surface to reconfigurable antenna technology," Proc. Active and Passive RF Devices Seminar, London, UK, February 2016, pp. 1-5.

[5] Subrt, L., Pechac, P.: "Intelligent walls as autonomous parts of smart indoor environments," IET Commun., 2012, 6, (8), pp. 1004-1010.

[6] Gustafsson, M., Karlsson, A., Pontes Rebelo, A.P., et al.: "Design of frequency selective windows for improved indoor outdoor communication,” IEEE Trans. Antennas Propag., 2006, 54, (6), pp. 18971900.

[7] Ch. Liaskos, Sh. Nie, A. Tsioliaridou, A. Pitsillides, S. Ioannidis, and I. Akyildiz. "A new wireless communication paradigm through software- controlled metasurfaces." IEEE Commun. Soc, vol. 56, no. 9, pp. 162169,2018

[8] G. I.Kiani, K. L. Ford, L. G. Olsson, K. P. Esselle, and Ch. J. Panagamuwa. "Switchable frequency selective surface for reconfigurable electromagnetic architecture of buildings. "IEEE Trans. Antennas Propagat., vol.58, no. 2, pp. 581-584, 2009

[9] J. Roberts, J. M. Rigelsford, and K. L. Ford. "Diffraction from Frequency Selective Surfaces for secure building applications." In 2012 6th European Conference on Antennas and Propagation (EUCAP), pp. 23882391. IEEE, 2012.

[10] Weiwei, W., Xi, C., Qi, F., et al.: "A measured FSS radome with two absorptive bands separated by one passband," Proc. 11th European Conf. Antennas Propagation (EUCAP), Paris, France, 2017, pp. 1118-1121

[11] Sangeetha, B., Gulati, G., Nair, R.U., et al. "Design of airborne radome using Swastika-shaped metamaterial-element based FSS," Proc. IEEE Annual India Conf. (INDICON), Bangalore, India, December 2016, pp. $1-4$.

[12] Ch. Liaskos, N. Shuai, T. Ageliki, P. Andreas Sotiris Ioannidis, and A. Ian "A new wireless communication paradigm through softwarecontrolled metasurfaces," IEEE Communications Magazine, vol. 56, no. 9, 2018, pp.162-169.

[13] W. Chen, C. A. Balanis and C. R. Birtcher., "Checkerboard EBG Surfaces for Wideband Radar Cross Section Reduction, " IEEE Trans. Antennas Propagat., vol. 63, no. 6, pp. 2636-2645, 2015.

[14] F. Samadi, M. Akbari, M.R. Chaharmir, and A. Sebak., "Scatterer Surface Design for Wave Scattering Application," IEEE Trans. Antennas Propagat., vol. 67, no. 2, pp.1202-1211, 2019. 